\title{
Crystals and Quasicrystals: A Continuum Model`
}

\author{
Charles Radin \\ Mathematics Department, University of Texas, Austin, TX 78712, USA
}

\begin{abstract}
We construct the first model of particles in the plane with completely symmetric, short range, two body interactions which has quasiperiodic, but no periodic, ground states.
\end{abstract}

\section{Introduction}

It is a problem of fundamental importance [1-5] to determine why, at low temperature, real matter strongly tends to have crystalline symmetry at the molecular level. The recent discovery [6] of quasicrystalline matter gives further impetus to understand to what extent, and especially by what mechanism, low temperature prescribes the symmetry of configurations of many interacting particles.

Using a grand canonical ensemble for several particle species, the low temperature distribution is concentrated on configurations with low value of $\left(e-\Sigma_{j} m_{j} d_{j}\right)$, where $e$ is the energy density and $m_{j}$ (respectively $d_{j}$ ) is the chemical potential (respectively particle density) of the $j$ th species. The symmetry of these configurations is the matter at issue.

Results on this "crystal problem" [7-23] have concentrated on classical mechanical models, mostly lattice gas models. The problem is essentially solved for one dimension, both for lattice gas [19] and continuum [20] models. Among two dimensional models a class with highly symmetric interactions is known $[15,21,23]$ to have periodic ground states. In contrast, recent results [21,23] have exhibited lattice gas models with no periodic ground states.

Using these recent lattice gas models we will construct the first model of particles in the plane (i.e. a continuous model) with completely symmetric, short range, two body interactions which has quasiperiodic, but no periodic, ground states. This will require that we also significantly enlarge the class of continuous models known to have periodic ground states.

\footnotetext{
* Supported in part by NSF Grant No. DMS-8501911
} 


\section{Definitions and Notation}

A (doubly) periodic configuration of particles in the plane is a "ground state" if it achieves the minimum value for $\left(e(C)-\Sigma_{j} m_{j} d_{j}(C)\right)$ amongst all periodic configurations $C$; the $m_{j}$ are fixed; see [20]. (From now on energy will mean potential energy, as kinetic energy is trivially minimized separately.) A configuration is "quasiperiodic" if, for any $\varepsilon>0$, with the exception of the part of the configuration in some area of relative density less than $\varepsilon$, the configuration is (doubly) periodic; the smaller the $\varepsilon$, the larger the period.

Throughout this paper we use a fixed coordinate system in the (complex) plane. In particular we will often refer to the unit triangular lattice $\{m \exp (i \pi / 3)+n \mid m$, $n=0, \pm 1, \pm 2 \ldots\}$, and we denote such points by their coordinates; $(m, n)$ stands for $m \exp (i \pi / 3)+n$.

\section{The Models}

We start with Berger's fundamental result [24] - an example of $N$ unit square "tiles" with top edges parallel to the real axis, copies of which, using translation alone and with given matching rules (which describe which edges may abut, corner to corner) can tile the plane but only quasiperiodically, not periodically (nor even periodically in a single direction.) (Since Berger's work various persons, e.g. Ammann [25], Penrose [25] and Robinson [25, 26], have produced examples with lower values of $N$; the lowest value I am confident of is $N=16$, due to Ammann [27,28].)

Using any such example we construct $N$ regular hexagonal tiles of edge length 3 , one hexagon for each of the $N$ squares. Fixing the hexagons with two edges always parallel to the real axis, we impart to the top (respectively bottom) edge of each hexagon the matching rules of the top (respectively bottom) edge of the corresponding square, and to the upper left (respectively lower right) edge the matching rules of the left (respectively right) edge of the corresponding square. Finally we allow any upper right edge to abut any lower left edge.

It is easy to see that, with the given matching rules, translations of copies of the hexagons may tile the plane but only quasiperiodically, not periodically.

Next we define 19 different "types" of "atom" for each hexagonal "molecule" as follows. Centering one of the $N$ hexagons on the point $(0,0)$, the 19 lattice points covered by the hexagon define the 19 atom types, each one henceforth labelled by the type of molecule it is associated with and by its defining coordinates with respect to the center of that molecule. For example $(3 ;(2,0))$ represents the atom type associated with the top right corner of the third type of hexagon. So we have $19 \mathrm{~N}$ different types of atoms. Arbitrary numbers of each of these atoms will eventually be allowed to be positioned independently in the plane, with two body interactions defined as follows.

The interaction between two atoms will depend only on their types and their separation; for each pair of types the interaction will be one of the following three functions of their separation $r$.

$$
V_{1}=\left\{\begin{array}{l}
+\infty, \quad 0 \leqq r<1 \\
(r-1.15) /(0.15), \quad 1 \leqq r \leqq 1.15 \\
0, \quad 1.15<r
\end{array}\right.
$$




$$
\begin{aligned}
& V_{2}=\left\{\begin{array}{l}
+\infty, \quad 0 \leqq r<1 \\
-4(r-1) / 15, \quad 1 \leqq r \leqq 1.15 \\
(r-7 / 4) / 15, \quad 1.15<r \leqq 7 / 4 \\
0, \quad 7 / 4<r,
\end{array}\right. \\
& V_{3}=\left\{\begin{array}{l}
+\infty, \quad 0 \leqq r<1 \\
0, \quad 1 \leqq r .
\end{array}\right.
\end{aligned}
$$

For each given atom type A (e.g. $(3 ;(2,0)))$ we must define through which of these interactions such an atom interacts with each other atom type $\mathrm{B}$. To do this, place $\mathrm{A}$ at the point in the plane with A's defining coordinates (i.e. $(2,0))$ and place one of each of the other 18 atom types associated with A's molecule (call this molecule $T$ ) at its coordinates. We now place other atoms near these, again in groups of 19 associated with molecules, as follows. The group of 19 atoms associated with tile $W$ may occupy the 19 lattice sites centered at $(5,-2)$, each at the position which is the translation by $(5,-2)$ of its coordinates, if and only if the bottom edge of tile $W$ may abut the top edge of tile $T$ by the matching rules. Similarly, the 19 atoms associated with tile $U$ may occupy the lattice sites centered at $(2,3)$ if and only if the lower left edge of $U$ may abut the upper right edge of $T$. Continue for sets centered at $(-3,5)$, $(-5,2),(-2,-3)$ and $(3,-5)$. Then atom $A$ interacts through $V_{1}$ with an atom of type B if and only if B appears at distance 1 from (that first) A in any of the above allowed constructions. Atoms $\mathrm{A}$ and $\mathrm{B}$ interact through $V_{2}$ if and only if $\mathrm{B}$ appears at distance $3^{1 / 2}$. Otherwise $A$ and $B$ interact through $V_{3}$. The chemical potentials for the $19 N$ atom species will have a common value $m \geqq 0$.

Now we allow arbitrarily many of each atom type to independently occupy positions in the plane (not just lattice sites.) For any (doubly) periodic (locally finite) configuration $C$ the energy density $e(C)$ and particle density $d(C)$ can be computed. We will show that in a ground state atoms automatically form hexagonal molecules, which can effectively be thought of as interacting so as to form a nonperiodic array. Since the atoms have a hard core, the hexagonal molecules (with atoms at the corners and in the interior) do not actually form a tiling but are separated and shifted in a simple way from a tiling.

Theorem 1. The infimum $f$ of $(e(C)-m d(C))$ over periodic configurations $C$ is not attained at any periodic $C$, but is attained at some quasiperiodic configuration.

Before we begin the proof we need to analyze a simpler system of one species of "new particles" with interaction $V_{1}+V_{2}$ and chemical potential $m$. The following is of independent interest.

Theorem 2. Assume one species of particle in the plane with chemical potential $m^{\prime} \geqq 0$ and interaction $V^{\prime}(r)$ satisfying

$$
V^{\prime}(r)=+\infty, \quad 0 \leqq r<1,
$$

$V^{\prime}(r)$ is strictly increasing on the interval $[1, c]$,

where $c$ is some fixed number, $3^{1 / 2}<c<1.9$,

$$
\begin{aligned}
& V^{\prime}(r)=0, c \leqq r, \\
& V^{\prime}(1)=-1, \\
& V^{\prime}(r) \geqq-0.04, \quad 1.15 \leqq r \leqq c .
\end{aligned}
$$


Then the infimum $f^{\prime}$ of $\left(e(C)-m^{\prime} d(C)\right)$ over periodic configurations $C$ is attained at the $C$ consisting of the unit triangular lattice.

Proof. We begin with a lemma.

Lemma. The minimum energy of interaction of a single particle $P$ with arbitrarily many other particles, all pairs with separation at least 1 , is obtained when $P$ interacts with exactly twelve particles; six at distance 1 , six at distance $3^{1 / 2}$.

Proof of the Lemma. By compactness there exists a configuration $C^{\prime}$ achieving the minimum interaction energy with $P$. From the separation restriction $C^{\prime}$ cannot contain more than six particles within distance 1.15 of $P$. Using (3) and the separation restriction a crude area argument shows that $P$ can interact with at most 24 particles. Therefore using (5) $C^{\prime}$ must have six particles within distance 1.15 of $P$. From (2) these six must actually be distance 1 from $P$. Then using the range of $V^{\prime}$ it follows that $P$ can interact with at most six more particles, one in each "valley" between pairs of those nearest to $P$. Finally, again from (2) these second six particles must be distance $3^{1 / 2}$ from $P$.

Now note that in any periodic configuration $C$

$$
\left(e(C)-m^{\prime} d(C)=\left(\frac{\frac{1}{2} \Sigma_{j} E\left(P_{j}\right)-m^{\prime} N}{N}\right)\left(\frac{N}{A}\right),\right.
$$

where $\left\{P_{j}\right\}$ are the particles in some unit cell of $C, E\left(P_{j}\right)$ is the energy of interaction of $P_{j}$ with all other particles in $C$ and $N$ (respectively A) is the number of particles in (respectively the area of) the unit cell. Using the lemma, the first (negative) factor on the right-hand side of (6) is minimized and using [29] the second (positive) factor is maximized, when $C$ is the unit triangular lattice. This proves Theorem 2.

Proof of Theorem 1. The energy between any pair of atoms at given positions cannot be lower than the energy between a pair of new particles at those positions. So $f \geqq f^{\prime}$. However by using a nonperiodic tiling $C^{\prime \prime}$ it is possible to place the atoms so as to make $e\left(C^{\prime \prime}\right)-m d\left(C^{\prime \prime}\right)=f^{\prime}$, so $f=f^{\prime}$. We will now prove by contradiction that there is no periodic configuration $C$ such that $e(C)-m d(C)=f$. Assume $C$ is such a periodic configuration. By the same argument as in Theorem 2 we see that the positions of the particles in $C$ form a unit triangular lattice with every pair of atoms separated by 1 interacting through $V_{1}$ and every pair separated by $3^{1 / 2}$ interacting through $V_{2}$. We have thus reduced the problem to that of a lattice gas version of the model. The last step is then very similar to that in [23], so we only outline the argument. We show that $C$ can be decomposed into disjoint "molecular" groups of 19 atoms in the same spatial relationships as in their definitions (with the possible exception that all the molecules may be rotated and/or reflected in the same way), and the molecules obey the matching rules. This contradicts the periodicity of $C$ and completes the proof.

\section{Stability}

Though we could generalize the above interactions to some extent, we do not know whether or not every sufficiently small change in the interactions preserves the 
qualitative result. One way to attack this stability problem, at least for lattice gas models, is by means of the Fraction Space introduced in [21]. For simplicity we will work with the two-dimensional triangular lattice, but the ideas generalize easily. Let $v_{1}, v_{2}$ and $v_{3}$ be three unit vectors in the lattice, pairwise $120^{\circ}$ apart. Assume $N$ species of particles can occupy each lattice site, with nearest neighbor interactions in the directions of the $v_{j}$. For each doubly periodic configuration $C$ of particles associate the $\left(3 N^{2}+N\right)$-component vector $f(C)$ whose components are defined as follows. The first $N$ components are the frequencies with which each of the $N$ species appears in $C$. The next $N^{2}$ components are the frequencies with which each of the $N^{2}$ pairs of species appear as nearest neighbors in $C$ in the direction of $v_{1}$ (the order in the pair is important); the next $N^{2}$ components similarly refer to the direction $v_{2}$ and the last $N^{2}$ to $v_{3}$. It is easy to check that the closure $\bar{F}$ of $F=\{f(C) \mid C$ is periodic $\}$ in $\mathbb{R}^{3 N^{2}+N}$ is bounded and convex. The supporting hyperplanes to $\bar{F}$ naturally correspond to the possible nearest neighbor interactions, together with chemical potentials, and the exposed points of $\bar{F}$ represent certain features of the ground states. If, as seems quite possible, $\bar{F}$ is a polyhedron, this would imply a great deal of stability in the crystal problem. For instance, converting the lattice gas version of the main example in this paper into a nearest neighbor model (by defining new lattice sites in place of sets of three original lattice sites as in [19]), the supporting hyperplane corresponding to the interaction contains a unique exposed point in $\bar{F} / F$. If $\bar{F}$ were known to be polyhedral we could vary the interaction (hyperplane) while preserving the nonperiodicity of the ground state. We note that the corresponding polyhedron problem for one dimensional models has an immediate positive solution for all $N$ from [19].

Acknowledgements. It is a pleasure to acknowledge useful conversations with Clifford Gardner and Robert Maier.

\section{References}

1. Anderson, P. W.: Basic notions of condensed matter physics. pp.11-15 Menlo Park: Benjamin/Cummings 1984

2. Brush, S. G.: Statistical physics and the atomic theory of matter. p. 277 Princeton: Princeton University Press 1983

3. Simon, B.: Fifteen problems in mathematical physics. In: Perspectives in mathematics: Anniversary of Oberwolfach 1984. pp. 442-443 Basel: Birkhäuser Verlag 1984

4. Uhlenbeck, G. E.: An outline of statistical mechanics. In: Fundamental problems in statistical mechanics II. p. 16 Cohen, E.G.D. (ed.) New York: Wiley 1968

5. Uhlenbeck, G. E.: Summarizing remarks. In: Statistical mechanics; Foundations and applications. p. 581 Bak, T. A. (ed). New York: Benjamin 1967

6. Schechtman, D., Blech, I., Gratias, D., Cahn, J. W.: Metallic phase with long-range orientational order and no translational symmetry. Phys. Rev. Lett. 53, 1951-1953 (1984)

7. Kunz, H.: The one-dimensional classical electron gas. Ann. Phys. (N.Y.) 85, 303-335 (1974)

8. Ventevogel, W. J.: On the configuration of a one-dimensional system of interacting particles with minimum potential energy per particle. Physica (Utrecht) 92A, 343-361 (1978)

9. Ventevogel, W. J., Nijboer, B. R. A.: On the configuration of systems of interacting particles with minimum potential energy per particle. Physica (Utrecht) 98A, 274-288 (1979) 
10. Ventevogel, W. J., Nijboer, B. R. A.: On the configuration of systems of interacting particles with minimum potential energy per particle. Physica (Utrecht) 99A, 569-580 (1979)

11. Gardner, C. S., Radin, C.: The infinite-volume ground state of the Lennard-Jones potential. J. Stat. Phys. 20, 719-724 (1979)

12. Hamrick, G. C., Radin, C.: The symmetry of ground states under perturbation. J. Stat. Phys. 21, 601607 (1979)

13. Heitmann, R. C., Radin, C.: The ground state for sticky disks. J. Stat. Phys. 22, 281-287 (1980)

14. Borden, B., Radin, C.: The crystal structure of the noble gases. J. Chem. Phys. 75, 2012-2013 (1981)

15. Radin, C.: The ground state for soft disks. J. Stat. Phys. 26, 365-373 (1981)

16. Wagner, H. -J.: Crystallinity in two dimensions: A note on a paper of C. Radin. J. Stat. Phys. 33, 523526 (1983)

17. Radin, C.: Crystalline symmetry and surface tension. Physica (Utrecht) 113A, 338-342 (1982)

18. Nicolò, F., Radin, C.: A first-order phase transition between crystal phases in the shift model. J. Stat. Phys. 28, 473-478 (1982)

19. Radin, C., Schulman, L.: Periodicity of classical ground states. Phys. Rev. Lett. 51, 621-622 (1983)

20. Radin, C.: Classical ground states in one dimension. J. Stat. Phys. 35, 109-117 (1984)

21. Radin, C.: Tiling, periodicity and crystals. J. Math. Phys. 26, 1342-1344 (1985)

22. Lieb, E., Kennedy, T.: To be published

23. Radin, C.: Crystals and quasicrystals: a lattice gas model. Phys. Lett. 114A, 381-383 (1986)

24. Berger, R.: The undecidability of the domino problem. Mem. Am. Math. Soc. 66 (1966)

25. Gardner, M.: Mathematical games. Sci. Am. 236, (1), 110-121 (1977)

26. Robinson, R. M.: Undecidability and nonperiodicity for tilings of the plane. Invent. Math. 12, 177209 (1971)

27. Robinson, R. M.: personal communication

28. Grünbaum, B., Shephard, G. C.: Tilings and patterns. To be published

29. Fejes-Tóth, L.: Úber einen geometrischen satz. Math. Z. 46, 79-83 (1940)

Communicated by J. Fröhlich

Received October 2, 1985; in revised form January 20, 1986 